\title{
Ballistic- and quantum-conductor carbon nanotubes: A reference experiment put to the test
}

\author{
M. Kobylko, ${ }^{1,2}$ M. Kociak, ${ }^{1}$ Y. Sato, ${ }^{3}$ K. Urita, ${ }^{3,4}$ A. M. Bonnot, ${ }^{5}$ A. Kasumov, ${ }^{1,6}$ Y. Kasumov, ${ }^{6}$ K. Suenaga, ${ }^{3}$ and C. Colliex ${ }^{1}$ \\ ${ }^{1}$ Laboratoire de Physique des Solides (LPS), CNRS/Université Paris-Sud UMR 8502, Bâtiment 510, Orsay, France \\ ${ }^{2}$ Laboratoire des Solides Irradiés (LSI), CEA/CNRS/Ecole Polytechnique UMR 7642, Ecole Polytechnique, Palaiseau, France \\ ${ }^{3}$ Nanotube Research Center, National Institute of Advanced Industrial Science and Technology (AIST), Tsukuba, Japan \\ ${ }^{4}$ Graduate School of Engineering, Nagasaki University, Nagasaki, Japan \\ ${ }^{5}$ Institut Louis Néel, CNRS/UJF UPR 2940, BP 166, Grenoble, France \\ ${ }^{6}$ Institute of Microelectronics Technology and High Purity Materials, Russian Academy of Sciences (IMT-RAS), \\ Chernogolovka, Moscow Region, Russia
}

(Received 13 March 2014; published 20 November 2014)

\begin{abstract}
We have performed electrical transport experiments on individual carbon nanotubes (CNTs) in situ in a transmission electron microscope using the liquid-metal contact method (LMC method), which consists of immersing a CNT placed on the apex of a metallic tip into a drop of liquid mercury $(\mathrm{Hg})$. In the literature, this method has been mostly employed without visualization ( ex situ) to show the ballistic- and quantum-conductance properties of different kinds of CNTs. We show that on the one hand the in situ LMC method is well suited to create low-resistance contacts with the CNTs but on the other hand the ballistic and quantum conductance measured by the ex situ LMC method is likely to give false positives for three reasons: (a) the CNTs are likely to be removed from the tip surface through contact with the $\mathrm{Hg}$, (b) occurring $\mathrm{Hg}$-tip surface nanocontacts are likely to be mistaken for quantum-conductor CNTs, and (c) occurring $\mathrm{Hg}$ nanomenisci are likely to be mistaken for ballistic-conductor CNTs. These findings have strong consequences for the interpretation of previously reported works.
\end{abstract}

DOI: 10.1103/PhysRevB.90.195431

PACS number(s): 73.63.-b, 73.63.Fg

\section{INTRODUCTION}

Carbon nanotubes (CNTs), discovered by Iijima [1], are a fascinating material. Depending on their diameter and helicity, they can be conductors or semiconductors with different band gaps [2]. Thanks to these properties they have a high potential to become the building blocks of a new electronics at nanoscale [3]. Recently, an important milestone has been reached by Shulaker et al. [4], who constructed the first complete fully programmable CNT computer based alone on CNT field effect transistors (CNT-FETs) and thus proved the suitability of CNTFET electronics for applications.

CNT-FET technology has the potential to become one order of magnitude more energy efficient than existing semiconductor technologies and thus to complement it in energy efficiency sensitive applications [5-8]. Notable technological achievements prior to the work of Shulaker et al. include the realization of CNT-FETs, logic gates, and stand-alone computational and storage circuit elements [9-14]. An important achievement is also the development of very large scale integration (VLSI) suitable fabrication methods which included overcoming challenges such as increasing the CNTFET density and preventing logic device malfunction due to mispositioned and/or metallic CNTs within CNT-FETs [15-17].

However, electronic applications of CNTs so far have only been making use of the semiconducting (respectively metallic) nature of CNTs (and their resulting contact Schottky barriers) but not exploiting possible ballistic conduction or conductance quantification effects [2]. Thus, the continuous technological progress notwithstanding, the field of application of CNTs as nanoelectronics building blocks remains limited as long as their electrical transport properties are not sufficiently understood. As a striking example and due to the difficulty of performing length-dependent conductivity measurements on well-characterized CNTs while limiting the influence of the environment (e.g., from the substrate), the question of whether multiwall CNTs (MWNTs) are ballistic $[18,19]$ or diffusive conductors $[20,21]$ has never been satisfactorily resolved.

One of the most important contributions in the debate on the ballistic or diffusive conduction in MWNTs is the famous experiment of Frank et al. [18] which showed (a) that MWNTs are room temperature ballistic conductors with electronic mean free paths (EMFPs) of several micrometers and (b) that the conductance of MWNTs is quantified in steps of $G=1 G_{0}=2 e^{2} / h$ [instead of $G=2 G_{0}$ as predicted by theory [2] for single-wall CNTs (SWNTs)]. This experiment was performed by using an elegant liquid-metal contact method (LMC method) consisting of immersing the MWNTs placed on the apex of a metallic tip into a liquid metal [usually mercury $(\mathrm{Hg})]$ and by supposing that in this way a mobile contact on the MWNT would be created [see Fig. 1(b)] which would allow one to measure the conductance versus length dependence of the immersed MWNT [see Figs. 1(a) and 1(c)].

This experiment has incited much similar work by several other groups [19,22-26] who considered the LMC method (or similar methods consisting of a cyclic immersion with no simultaneous visualization) also applicable on thin CNTs with micrometric lengths, i.e., single-wall CNTs (SWNTs) [23,25,26], double-wall CNTs (DWNTs) [22], and MWNTs $[19,24]$ with a small number of walls. The interest of this kind of CNTs lies in their micrometric length which allows one to test micrometric EMFPs and in their limited wall number which facilitates the study of interwall interactions as well as in the unambiguous identification of their chiral indices by electron diffraction [27]. 


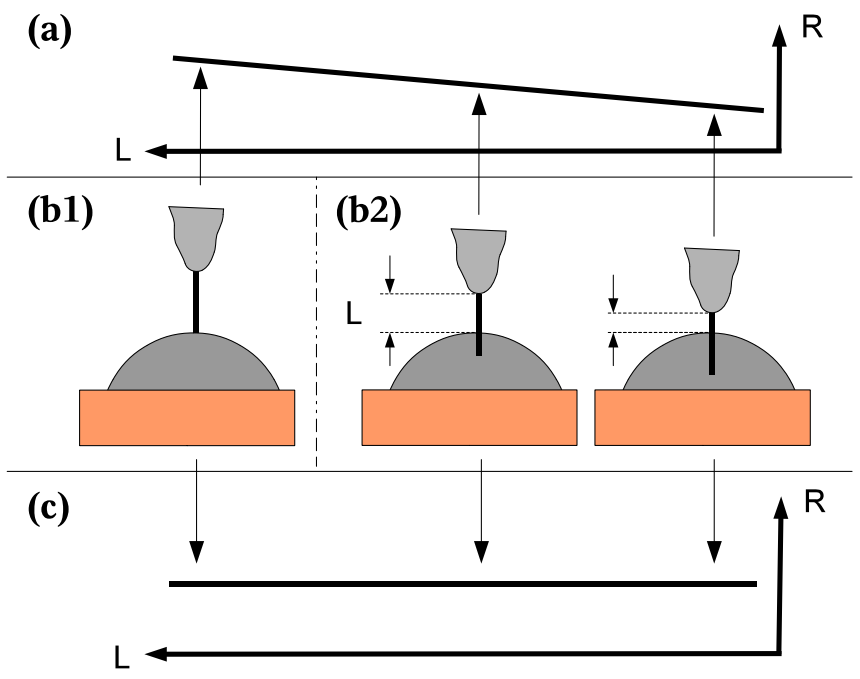

FIG. 1. (Color online) Illustration of the liquid-metal contact (LMC) method: (a) resistance-length dependence of diffusive CNTs, (b) schematic drawing of a CNT placed on a metallic tip being immersed into a drop of liquid metal, (c) resistance-length dependence of ballistic CNTs. In (b1), the resistance of the whole CNT is measured, whereas in (b2) only the resistance of the nonimmersed part of the CNT is measured. See text for more explanations.

In all these experiments, ballistic conductance was confirmed for the studied CNTs and EMPFs of the order of $10^{-6} \mathrm{~m}$ and even of the order of $10^{-5} \mathrm{~m}$ have been determined. Surprisingly, experiments performed with the liquid-metal method showed only once diffusive conduction of CNTs, namely by Poncharal et al. [28], and only on CNTs coated with a surfactant. To the best of our knowledge, diffusive conduction of pure CNTs has never been reported by groups using the liquid-metal contact or similar techniques, in contrast to other types of electrical transport experiments on individual CNTs [20,21].

In this paper, we first provide experimental evidence that (i) the in situ LMC method is well suited to create low-resistance contacts with CNTs and that (ii) the ballistic and quantum conductance measured by the ex situ LMC method is likely to give false positives for three reasons: (a) the CNTs are likely to be removed from the tip surface through contact with the $\mathrm{Hg}$, (b) occurring Hg-tip surface nanocontacts are likely to be mistaken for quantum-conductor CNTs, and (c) occurring $\mathrm{Hg}$ nanomenisci are likely to be mistaken for ballistic-conductor CNTs. In consequence, many previous experimental results claiming ballistic and quantum conductance in CNTs have to be reinterpreted. By showing that the conductance measurements performed with the LMC method are likely to belong to other objects than CNTs (e.g., to $\mathrm{Hg}$ nanomenisci), this paper also helps to understand the experimental measurement of a $1 G_{0}$ MWNT conductance in contradiction to the theoretical SWNT conductance of $2 G_{0}$.

\section{DESCRIPTION OF THE EXPERIMENT}

We have performed several series of in situ transport experiments on individual thin CNTs (MWNTs with roughly five walls or less) using the Hg LMC method. Our CNTs have been grown by two different CCVD methods using either $\mathrm{Co} / \mathrm{Ni}$ [29] or $\mathrm{Al} / \mathrm{Fe}$ catalysts [30] on nanometric tungsten (W) tips (obtained by electrochemical etching) and on AFM tips (metalized with a $20 \mathrm{~nm}$ thick $\mathrm{Nb}$ or Ti layer for better conductance).

The in situ transport experiments were performed inside a JEOL 2010F transmission electron microscope (TEM). We used two commercial nanomanipulator specimen holders: the JEOL EMZ8139T, which we combined with self-made electronics and software, and a Nanofactory [31] STM-TEM holder [32], suited with its own electronics and software. These in situ specimen holders allow electrical transport measurements with simultaneous visual information and mechanical control which is indispensable for this kind of experiment. Inside these specimen holders, the experimental geometry consists of two electrodes (one mobile, controlled by a piezoelectric ceramic, and one fixed) which face each other, one being the CNT-carrying tip and the other (the counterelectrode) a metallic tip carrying a $\mathrm{Hg}$ droplet (or in some of our experiments an Au tip). The apexes of both electrodes are in the field of view of the TEM.

\section{EXPERIMENTAL RESULTS AND DISCUSSION}

\section{A. The in situ liquid-metal contact method is well suited to create low-resistance contacts with CNTs}

The first part of our experimental study dealt with the question of whether the in situ LMC method (i.e., performed inside a TEM and thus with visualization) is well suited to create low-resistance contacts with CNTs in the configuration depicted in Fig. 1(b1), i.e., whether contacting the CNT with a liquid metal will result in a lower resistance contact than contacting it with a solid metal. Therefore, in some of our in situ experiments, we have replaced the $\mathrm{Hg}$ counterelectrode with an Au counterelectrode in order to measure the difference in the contact resistances between these two metals. We performed approximately 140 measurements on 9 CNTs brought into contact with $\mathrm{Hg}$ and $10 \mathrm{CNT}$ s brought into contact with $\mathrm{Au}$.

The resistance values were taken at low bias $(0.1 \mathrm{~V})$. Two sets of five tiplike samples as seen in Fig. 2 have been used respectively with the $\mathrm{Au}$ counterelectrode and the $\mathrm{Hg}$ counterelectrode. Three samples have been part of both sets, thereunder sample 1 and sample 2 referred to in Fig. 3 and Table I. Figure 3 shows histograms of the resistance

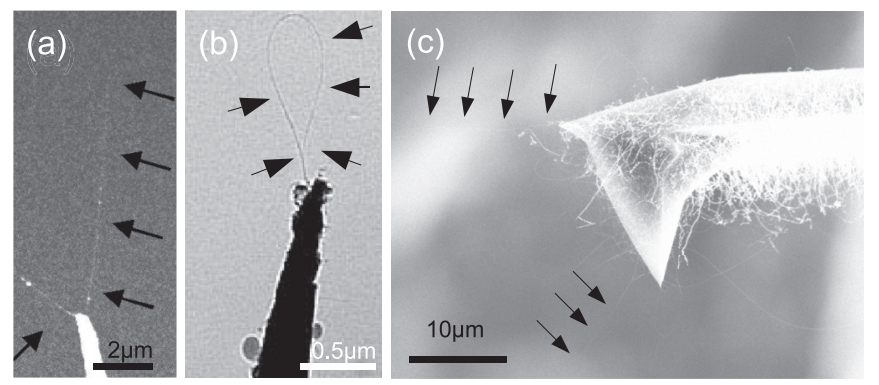

FIG. 2. CNTs on tips: (a) SEM image and (b) TEM image of CNTs on the apices of W tips, (c) SEM image of CNTs on the apex of an AFM tip. 

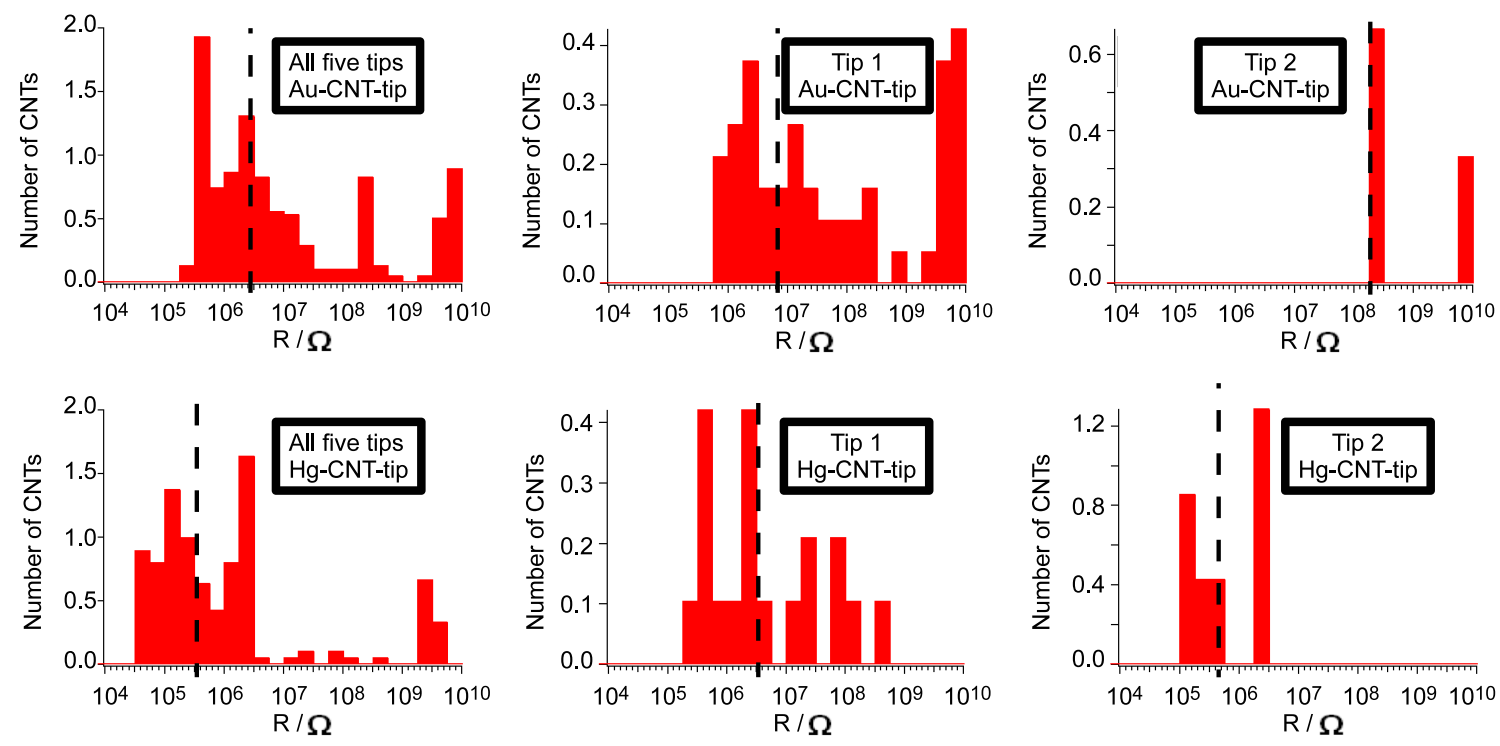

FIG. 3. (Color online) Histograms of Au/CNT-tip and Hg/CNT-tip low-bias contact resistance values. Note the logarithmic resistance scale. The area under the histograms gives the number of CNTs on the respective sample. Upper row: Au/CNT-tip resistance values. Lower row: $\mathrm{Hg} / \mathrm{CNT}$-tip resistance values. Left column: Resistance values for all five samples taken on $10 \mathrm{CNTs}$ (for Au) respectively $9 \mathrm{CNTs}$ (for Hg) in contact with different places on the $\mathrm{Au}$ (respectively $\mathrm{Hg}$ ) counterelectrode. Middle column: Resistance values for sample 1 taken on 3 CNTs (for $\mathrm{Au}$ ) respectively on $2 \mathrm{CNTs}$ (for $\mathrm{Hg}$ ) in contact with different places on the $\mathrm{Au}$ (respectively $\mathrm{Hg}$ ) counterelectrode. Right column: Resistance values for sample 2 taken on $1 \mathrm{CNT}$ (for $\mathrm{Au}$ ) respectively on $3 \mathrm{CNTs}$ (for $\mathrm{Hg}$ ) in contact with different places on the Au (respectively $\mathrm{Hg}$ ) counterelectrode. Dashed lines indicate the positions of the geometrical average resistances. Resistance values above $1 \mathrm{G} \Omega$ have not been considered in the calculation of the geometrical average resistances.

values for sample 1 , for sample 2 , and for all samples. These histograms of subsequent conductivity measurements have been normalized in such a way as to give each CNT the same statistical weight, taking into account that the number of conductivity measurements performed on each CNT differed between different CNTs. Hence, the area under the histograms is equal to the number of CNTs on the respective sample. The legend of Fig. 3 gives further information. Resistance values above $1 \mathrm{G} \Omega$ are not trustworthy because our measurement system has a leakage-current-induced detection limit between $1 \mathrm{G} \Omega$ and $10 \mathrm{G} \Omega$.

TABLE I. Comparison of Au/CNT-tip and Hg/CNT-tip lowbias contact resistance values. Left column: Geometrical average resistance of $10 \mathrm{CNTs}$ (for $\mathrm{Au}$ ) respectively $9 \mathrm{CNTs}$ (for $\mathrm{Hg}$ ) mounted on five different tips in contact with different places on the $\mathrm{Au}$ respectively $\mathrm{Hg}$ counterelectrode. Middle column: Geometrical average resistance of $3 \mathrm{CNTs}$ (for $\mathrm{Au}$ ) respectively $2 \mathrm{CNTs}$ (for $\mathrm{Hg}$ ) mounted on tip 1 in contact with different places on the $\mathrm{Au}$ respectively $\mathrm{Hg}$ counterelectrode. Right column: Geometrical average resistance of $1 \mathrm{CNT}$ (for $\mathrm{Au}$ ) respectively $3 \mathrm{CNTs}$ (for $\mathrm{Hg}$ ) mounted on tip 2 in contact with different places on the $\mathrm{Au}$ respectively $\mathrm{Hg}$ counterelectrode. Last row: Ratio of the respective $\mathrm{Au} / \mathrm{CNT}$-tip contact resistance to the $\mathrm{Hg} / \mathrm{CNT}$-tip contact resistance.

\begin{tabular}{lccc}
\hline \hline & $\overline{R_{\text {total }}} / \Omega$ & $\overline{R_{1}} / \Omega$ & $\overline{R_{2}} / \Omega$ \\
\hline $\mathrm{Au}$ & $2.7 \times 10^{6}$ & $6.8 \times 10^{6}$ & $1.8 \times 10^{8}$ \\
$\mathrm{Hg}$ & $3.3 \times 10^{5}$ & $3.4 \times 10^{6}$ & $4.4 \times 10^{5}$ \\
$\mathrm{Ratio}$ & 8.2 & 2.0 & $4.1 \times 10^{2}$ \\
\hline \hline
\end{tabular}

One notices that the resistances values in Fig. 3 are widely distributed which shows that the contact resistance value depends strongly on the place where the CNT touches the Au or $\mathrm{Hg}$ counterelectrode. One sees that the distributions of the $\mathrm{Hg} / \mathrm{CNT}$-tip low-bias contact resistances are shifted to the left in comparison to the respective distribution of the Au/CNT-tip low-bias contact resistances. This shift can be analyzed in several ways. First, one can look on the minimum resistances which are approximately $40 \mathrm{k} \Omega\left[=1 /\left(0.3 G_{0}\right)\right]$ for a $\mathrm{Hg} / \mathrm{CNT}$ tip system and approximately $250 \mathrm{k} \Omega\left[=1 /\left(0.05 G_{0}\right)\right]$ for a $\mathrm{Au} / \mathrm{CNT}$-tip system. Second, one can compare the peaks which dominate the resistance distributions. These are approximately $400 \mathrm{k} \Omega$, approximately $2 \mathrm{M} \Omega$, and approximately $200 \mathrm{M} \Omega$ for the Au/CNT-tip systems and approximately $100 \mathrm{k} \Omega$ and approximately $2 \mathrm{M} \Omega$ for the $\mathrm{Hg} / \mathrm{CNT}$-tip systems. Third, one can compare the average resistance values. Due to the wide distribution of the resistance values spanning several orders of magnitude, geometrical averages are more appropriate than arithmetic averages. Thus, the geometrical average resistances have been calculated according to Eq. (1):

$$
\bar{R}=10^{\left(\sum_{i=1}^{n} \log _{10} R_{i}\right) / n} .
$$

As they are not trustworthy, the resistance values above $1 \mathrm{G} \Omega$ have not been considered in the calculation of the geometrical average resistances. The geometrical average resistances are indicated in Fig. 3 as dashed lines. Their numerical values are shown in Table I. For sample 1, the geometrical average $\mathrm{Hg} / \mathrm{CNT}$-tip resistance is approximately 2 times smaller than the geometrical average Au/CNT-tip resistance. For sample 2, the geometrical average $\mathrm{Hg} / \mathrm{CNT}$-tip resistance is approximately $4.1 \times 10^{2}$ times smaller than the 
geometrical average $\mathrm{Au} / \mathrm{CNT}$-tip resistance. In total, the geometrical average $\mathrm{Hg} / \mathrm{CNT}$-tip resistance is 8.2 times smaller than the geometrical average Au/CNT-tip resistance. Fourth, one can compare not only the histograms in which each CNT has the same statistical weight as in Fig. 3, but also histograms in which each sample has the same statistical weight (not shown), or in which each measurement has the same statistical weight (not shown). Those results are qualitatively the same as these shown here.

In conclusion, the minimum resistances we have measured in situ at low bias were approximately $40 \mathrm{k} \Omega\left[=1 /\left(0.3 G_{0}\right)\right]$ for a $\mathrm{Hg} / \mathrm{CNT}$-tip system and approximately $250 \mathrm{k} \Omega$ $\left[=1 /\left(0.05 G_{0}\right)\right]$ for an Au/CNT-tip system. Independently of the statistical analysis technique, our results show clearly that $\mathrm{Hg}-\mathrm{CNT}$ contacts are on average between three and ten times less resistive at low bias than $\mathrm{Au}-\mathrm{CNT}$ contacts. Therefore we conclude that the in situ LMC method (i.e., performed in a TEM and thus with visualization) is well suited to create low-resistance contacts with the CNTs and, in consequence, also to measure the total conductance of the non-Hg-immersed part of the CNT in the configuration depicted in Fig. 1(b1).

\section{B. The ballistic- and quantum-conductance measurement} by the ex situ LMC method is likely to give false positives

The second part of our experimental study dealt with the question of whether the ex situ LMC method (i.e., not performed in a TEM and thus without visualization) is reliable in the two configurations (b1) and (b2) depicted in Fig. 1. For the LMC method to be reliable ex situ, it is important that the measured conductance can only be the conductance of the CNT and of nothing else. In the following, we will show by means of in situ LMC experiments that this necessary condition is not fulfilled.

\section{The LMC method with simultaneous in situ visualization}

In Fig. 4, we show the results of an in situ experiment realized using the common experimental protocol for ex situ experiments involving cyclic immersion of CNTs into the $\mathrm{Hg}$ and the representation of the measured conductance values in a histogram. Figure 4 shows the conductance vs time dependence while a sinusoidal voltage is applied to the piezoelectric ceramic. The conductance does not change in a continuous way (as would be the case for a diffusive conductor) but forms well-defined stable plateaus over up to about $300 \mathrm{~nm}$ length. We see that the conductance takes a higher value when the $\mathrm{Hg}$ meniscus touches a bigger part of the tip surface (right part of Fig. 4).

According to the criteria suggested in the original work of Frank et al. [18] and subsequently accepted in the literature [19,22-26], the same experimental results (if acquired in an ex situ experiment without visual control) would show that the CNT at the apex of the tip is a ballistic conductor (due to the stability of the plateaus) and that its conductance is quantified (due to the appearance of peaks in the histogram). One can therefore define a quantification step $G_{1}$ which is the conductance value of which the total measured conductance appears to always be an integer multiple. In the picture adopted by Frank et al. [18], $G_{1}$ would thus correspond to the conductance of a single CNT and measuring multiples of $G_{1}$ would signify that several CNTs are immersed in the $\mathrm{Hg}$ at the same time. The value of $G_{1}$ can be deduced from the histogram (see right side of Fig. 4). However, in the data set we show $G_{1}$ cannot be determined unambiguously due to the presence of only two peaks and its broadness. Two (mutually exclusive) values of $G_{1}$ are indeed possible. First, the quantification step $G_{1}$ could be $G_{1}=2.5 \ldots 3 G_{0}$ in which case the left two plateaus of Fig. 4 would correspond to a conductance of $G=1 G_{1}$ and the right two plateaus of Fig. 4 to a conductance of $G=2 G_{1}$. Second, the quantification step $G_{1}$ could be $G_{1}=1.0 G_{0}$ in which case the left two plateaus of Fig. 4 would correspond to a conductance of $G=3 G_{1}$ and the right two plateaus of Fig. 4 to a conductance of $G=5 G_{1}$. However, for our present study it is not the precise value of $G_{1}$ which is important but rather the fact that the value of $G_{1}$ is close to conductance quantum $G_{0}$ which has been identified as the quantification step of CNTs by Frank et al. [18]. Figure 4 thus unambiguously shows that the ex situ LMC method is likely to give false positives of ballistic transport properties, of conductance quantification, and of the conductance quantification step value. Note that the here presented histogram (despite showing only two peaks of which one is broadened) is nonetheless comparable to those from which conductance quantification of CNTs has been concluded $[18,33]$ as those were similarly broadened and sometimes did not even show two peaks as here but rather as little as one single peak.

\section{Reasons for the unreliability of the ex situ LMC method}

Figure 4 demonstrates that a measurement equivalent to that of Frank et al. [18] can be obtained without even a CNT present. In this section, we point out how this fact makes the LMC method unreliable in an ex situ experiment without simultaneous visualization.

The first reason is that (as we could observe in our in situ experiments) the CNTs on the tip surface are likely to be removed through contact with the $\mathrm{Hg}$. The experimental in situ setup permitted us to visualize CNTs immediately before and immediately after an immersion into the $\mathrm{Hg}$. Poncharal et al. [34] have already observed a cleansing effect of the $\mathrm{Hg}$ on the sample in low-magnification in situ experiments: removing graphitic particles and poorly connected CNTs by the $\mathrm{Hg}$. In our higher magnification in situ experiments we could see this effect more precisely (see Figs. S1 and S2 in the Supplemental Material [35] for two examples): after a $\mathrm{Hg}$ immersion, we often saw CNTs move, break, or disappear. Moving the CNTs can make them stick together or fall apart. The Hg also moves clusters of metallic and graphitic particles and sometimes deposits them on the CNTs. Sometimes, a $\mathrm{Hg}$ immersion can even remove parts of the tip surface layer together with the CNTs located on it. Thus, even if it is not always the case, already a number of $\mathrm{Hg}$ immersions as small as five has often a devastating effect on the CNTs at the apex. We conclude from this observation that, in general, a removal of the CNTs by the $\mathrm{Hg}$ (and thus the creation of an experimental configuration analogous to that depicted in Fig. 4) during ex situ experiments involving hundreds or thousands of $\mathrm{Hg}$ immersions (like in the original work of Frank et al. [18]) is extremely likely. 


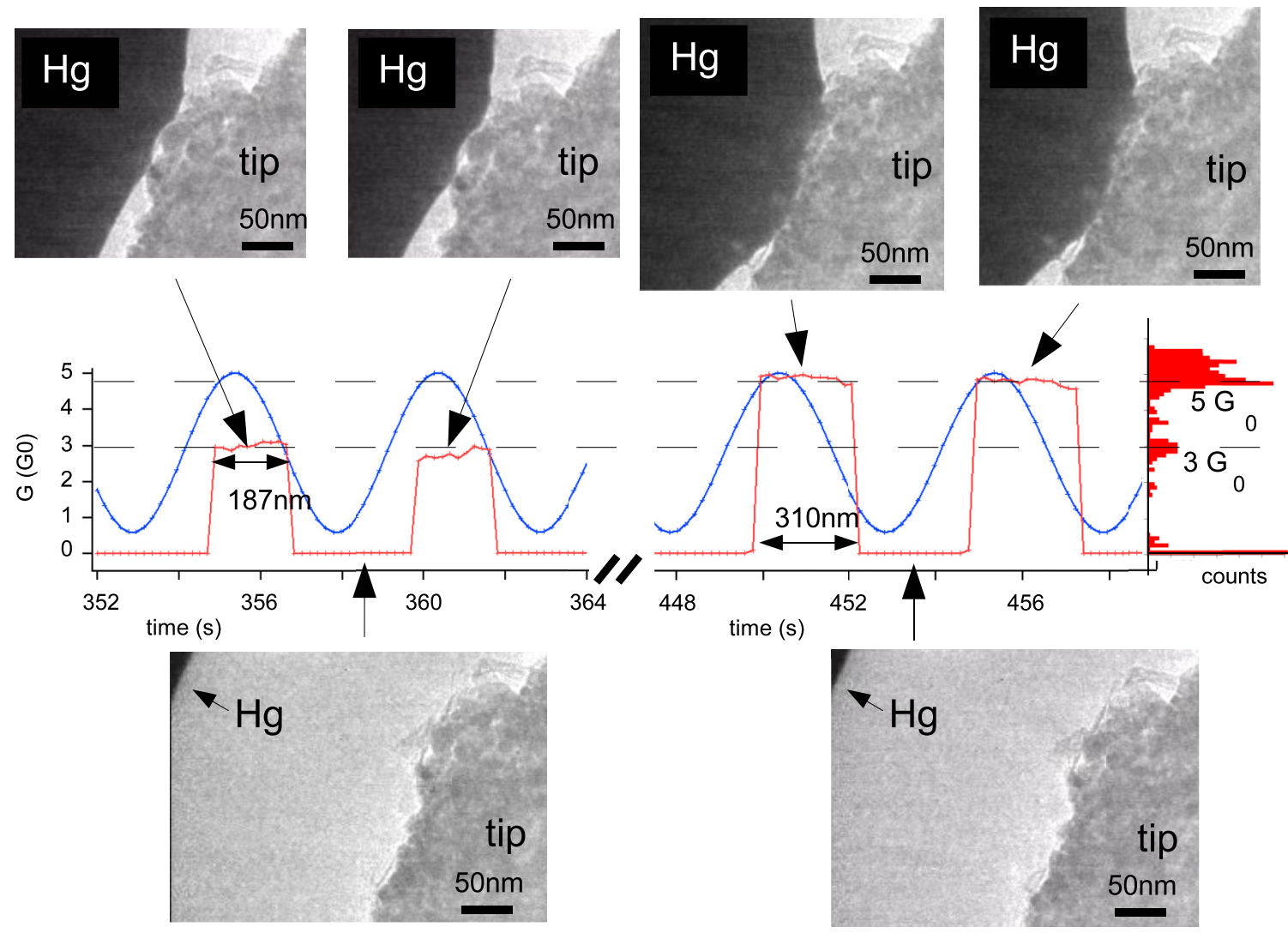

FIG. 4. (Color online) Upper part: the conductance vs time dependence, acquired in situ during cyclic immersion of a tip with CNTs at the apex into the $\mathrm{Hg}$ according to the common experimental protocol for ex situ experiments, meets the criteria for ballistic transport and conductance quantification [red: conductance, blue: sample displacement (amplitude: $220 \mathrm{~nm}$ )]; lower part: only visual control can tell us that the transport measurement is not being performed on CNTs but on several Hg nanomenisci sticking to the tip surface, the CNTs having been removed by the $\mathrm{Hg}$ during previous immersions. The length of the conductance plateaus as expressed in nm is defined as the position of the sample at the beginning of the plateau minus the position of the sample at the end of the plateau.

The second reason is that (in an experimental configuration without CNTs) $\mathrm{Hg}$ menisci are likely to be mistaken for ballistic conductors. The reason for that is that (as observed in our in situ experiments) they follow the mechanical movement of the (previously CNT-carrying) tip over hundreds of nanometers and that (as also observed in our in situ experiments) their conductance does not depend on their length. (The last point indicates that it is rather the resistance of the $\mathrm{Hg}$-tip contact that we measure rather than the resistance of the $\mathrm{Hg}$ meniscus. This point will be discussed in Sec. III C.) These two observations are already shown in Fig. 4 in a dynamical setting (periodically oscillating mechanical displacement). Figure 5 confirms these observations in a more statical setting. It shows a $\mathrm{Hg}$ meniscus sticking to a tip apex carrying no CNTs. During retraction and approach of the tip to the $\mathrm{Hg}$ droplet of about $100 \mathrm{~nm}$, the $\mathrm{Hg}$ meniscus follows the tip. Note that a tip displacement of about $100 \mathrm{~nm}$ is not unusually short for ex situ experiments carried out with the liquid-metal contact method [18]. During this mechanical manipulation, the conductance varies but the variation is uncorrelated to the mechanical displacement. In addition, the value of the conductance is between $0.5 G_{0}$ and $1 G_{0}$ which is very close to the conductance value of $1 G_{0}$ which has been assigned by Frank et al. [18] to ballistic CNTs. Such experimental results, if acquired in an ex situ experiment without visual control, would be likely to suggest a ballistic CNT at the apex of the tip (in particular at the much shorter time scales and the dynamical setting used in the original work of Frank et al. [18], which would not make the conductance variation as apparent as in the statical setting of Fig. 5). In reality, there is not even a CNT present, and one measures only the conductance of the $\mathrm{Hg}$ meniscus which follows the tip.

The third reason is that $\mathrm{Hg} / \mathrm{tip}$-surface nanocontacts are likely to be mistaken for CNTs in an ex situ LMC experiment, as they are likely to have conductance values close to the conductance quantum $G_{0}$ (see Fig. 4 and Fig. 5). In Sec. III C, the origin of this conductance value is discussed.

Due to the three above-mentioned reasons, one can assure the presence of CNTs on the apex of the tip and the correct interpretation of the experimental results only by simultaneous in situ visualization. In Fig. 4, no CNT is present at the tip of the sample due to before-mentioned removal of the CNTs during previous $\mathrm{Hg}$ immersions. Instead of the CNT conductance, we measure the conductance of the $\mathrm{Hg}$ nanocones and the tip surface covered by catalytic particles and graphitic layers. The illusion of ballistic transport arises from the fact that the $\mathrm{Hg}$ meniscus follows the retracting tip and that the conductance is most likely determined by the $\mathrm{Hg}$-tip contact rather than the 
(A)
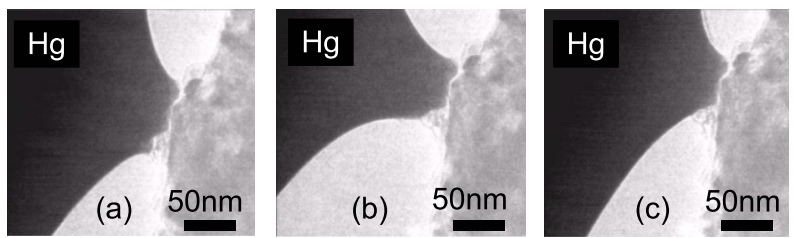

(B)

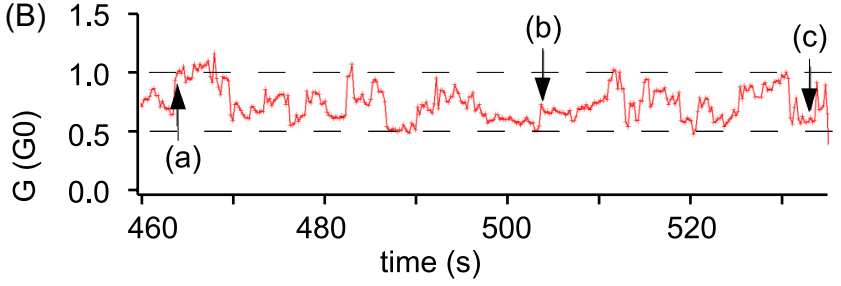

FIG. 5. (Color online) A Hg meniscus sticking to the apex of the tip. (A) Visualization. (B) Conductance vs time dependence. (a), (b), and (c) designate the moments in (B) at which the corresponding images in (A) were taken. The sample displacement between (a) and (b), and between (b) and (c), is monotonic. During retraction and approach of the tip to the $\mathrm{Hg}$ droplet of about $100 \mathrm{~nm}$, the $\mathrm{Hg}$ meniscus follows the tip. The conductance varies but the variation is uncorrelated to the mechanical displacement.

conductance of the $\mathrm{Hg}$ meniscus itself. The fact that at almost each $\mathrm{Hg}$ immersion the same kind of contact as at the previous immersion is formed, and that the same contact has always the same conductance, is responsible for the occurrence of peaks in the conductance histogram. The existence of a conductance value $G_{1}$, on whose multiples these peaks are centered, creates the illusion of quantification of conductance in steps of $G_{1}$.

As confirmed by our in situ observations, Fig. 4 depicts a scenario which is extremely likely to happen (but impossible to detect) in ex situ LMC experiments (i.e., without simultaneous visualization) on CNT-carrying tips, in particular in the oscillating and highly repetitive conditions (hundreds to thousands of oscillations) of the experiments of Frank et al. [18] and similar work [19,22-24]. Therefore, the ex situ LMC method must be considered unreliable in both configurations (b1) and (b2) depicted in Fig. 1.

\section{Origin of the measured quantum conductance}

In addition to showing the unreliability of the liquidmetal contact method, our in situ experiments show that the conductance of a $\mathrm{Hg}$ nanomeniscus contacted to the tip surface across catalytic and graphitic particles can be close to the quantum conductance $G_{0}$. In order to answer the question if this conductance value can be attributed to the $\mathrm{Hg}$ nanomeniscus itself, we estimated its expected conductance value in two different ways. First, we estimated the conductance of three $\mathrm{Hg}$ nanomenisci shown in Figs. 5 and 4 using the classical macroscopic concept of resistivity. From the definition of resistivity $\rho$, one can easily deduce Eq. (2) for the classical conductance $G_{\text {class }}$ of a homogenous cone with the smaller radius $r_{1}$, the bigger radius $r_{2}$, and the length $L$ :

$$
G_{\text {class }}=\frac{\pi r_{1} r_{2}}{\rho L}
$$

These so calculated conductance values of the $\mathrm{Hg}$ nanomenisci, shown in Table II, are between $10^{2}$ and $10^{4}$ times the measured ones. Second, we estimated the conductance of the same $\mathrm{Hg}$ nanomenisci by assuming them to be narrow ballistic quantum conductors with a limited number of transverse electronic modes. The Fermi wavelength $\lambda_{F}$ of $\mathrm{Hg}$ has been roughly approximated with the free-electron-model value [36] $\lambda_{F}=[(8 \pi) /(3 n)]^{1 / 3}$ ( $n$ being the number of electrons per volume unit which gives $\lambda_{F}=0.47 \mathrm{~nm}$ for $\mathrm{Hg}$ ). According to Datta [37], the rough number $M_{\text {trans }}$ of the transverse electronic modes in a narrow cylinder with the radius $r_{1}$ and the length $L$ is given by Eq. (3) ( $\lfloor x\rfloor$ being the biggest integer which is not bigger than $x$ ):

$$
M_{\text {trans }}=\left\lfloor\frac{\pi r_{1}^{2}}{\left(\lambda_{F} / 2\right)^{2}}\right\rfloor .
$$

The conductance $G_{\text {quant }}$ of a ballistic quantum conductor with this number of modes is then simply given by Eq. (4) [37]:

$$
G_{\text {quant }}=G_{0} M_{\text {trans }} .
$$

These so calculated conductance values of the $\mathrm{Hg}$ nanomenisci, shown in Table II, are between $10^{3}$ and $10^{5}$ times the measured ones. As the quantum-conductor estimation delivers values at least one order of magnitude higher than the classical estimation, one usually would conclude that these $\mathrm{Hg}$ nanomenisci should theoretically behave as classical conductors. However, regardless of whether we assume them to be classical conductors or to be quantum conductors, the estimated resistance values of the $\mathrm{Hg}$ nanomenisci are several orders of magnitude lower than the measured ones. Hence, we conclude that the measured resistance cannot come from the $\mathrm{Hg}$ nanomenisci if these can be described either as classical conductors with the same resistivity as bulk $\mathrm{Hg}$ or as narrow ballistic conductors with the same transverse electronic mode density as bulk $\mathrm{Hg}$. We rather suppose that the tipsurface-covering layer of catalytic particles and graphite is not homogeneously conducting and that the measured resistance of the $\mathrm{Hg}$ nanomenisci could potentially be attributed to the percolation resistance of this layer.

This hypothesis is supported by the experimental observation of forty different $\mathrm{Hg}$ nanomenisci sticking to about ten different places on the tip and the measurement of their conductance. Figure 6 shows some examples of these $\mathrm{Hg}$ nanomenisci with their respective conductance values. The $\mathrm{Hg}$ nanomenisci sticking to the same place nearly always had the same conductance. Where occasionally this was not the case, we attribute this to a slight change of the sticking surface whose exact observation is impossible for geometrical reasons. In contrast, comparable $\mathrm{Hg}$ nanomenisci sticking to different places had very different conductance values. For about half of the sticking places the conductance was ranging from $0.3 G_{0}$ to $3.0 G_{0}$, however always staying of the order of $G_{0}$; for the other half the conductance was $0 G_{0}$. We therefore assume that only a randomly distributed fraction of the tip-surface covering layer of our samples is conductive leading to a percolation type of conductance and that the conductance of a $\mathrm{Hg}$-nanomeniscus-to-tip contact depends among others on how many of these conductive spots the $\mathrm{Hg}$ nanomeniscus touches. We also conclude that, contrary to expectations, direct 
TABLE II. Smaller radius, bigger radius, length, classically estimated, quantally estimated, and experimentally measured conductance of different $\mathrm{Hg}$ menisci. (Especially for Fig. 4, the geometrical values are very imprecise and should be rather understood in terms of orders of magnitude.)

\begin{tabular}{lrrrrrc}
\hline \hline Meniscus & $r_{1} / \mathrm{nm}$ & $r_{2} / \mathrm{nm}$ & $L / \mathrm{nm}$ & $G_{\text {class }} / G_{0}$ & $G_{\text {quant }} / G_{0}$ & $G_{\exp } / G_{0}$ \\
\hline Fig. 5 (middle) & 25 & 25 & 50 & $\sim 530$ & $\sim 36000$ & $0.5 \ldots 1.0$ \\
Fig. 4 (left) & 10 & 30 & 10 & $\sim 1300$ & $\sim 5700$ & 3 \\
Fig. 4 (right) & 150 & 250 & 30 & $\sim 53000$ & $\sim 1300000$ & 5 \\
\hline
\end{tabular}

CCVD growth of CNTs on metal samples does not necessarily result in low-resistance contacts.

\section{Is the in situ LMC method suited to measure the conductance-length dependence of CNTs?}

In addition to the question of whether the ex situ LMC method is reliable, we have also investigated whether the in situ LMC method is suited to measure the conductance-length dependence of CNTs in the configuration depicted in Fig. 1(b2) if one manages to rule out the possibility of the removal of the CNTs from the tip surface and the measurement of the $\mathrm{Hg}$ nanomenisci and nanocontacts (instead of the CNT) in a specially designed in situ experiment. Note that proof for ballistic and quantum conductance in CNTs from such experiments has to our best knowledge never been reported.
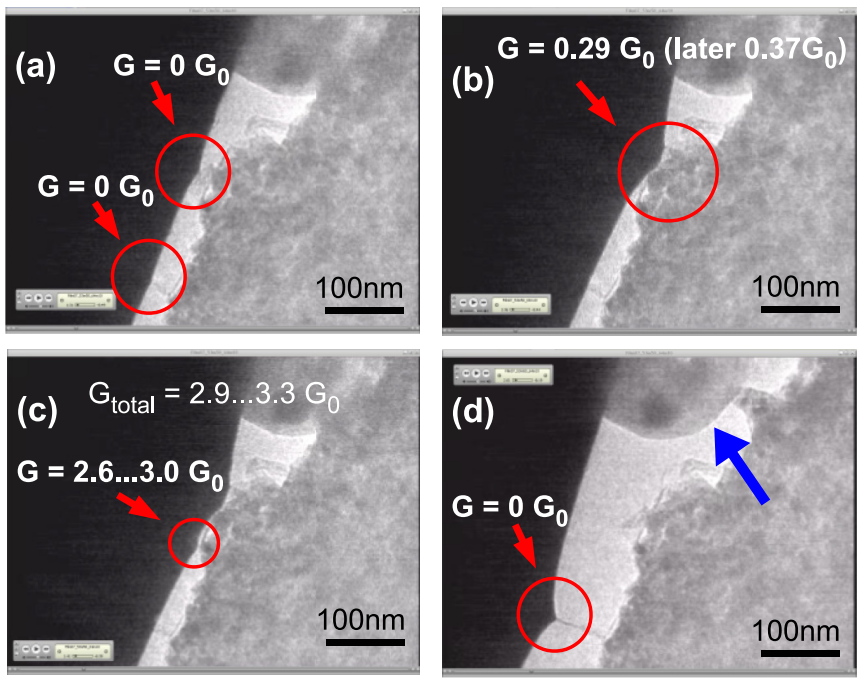

FIG. 6. (Color online) Examples of $\mathrm{Hg}$ nanomenisci sticking to different places on the tip surface $(\mathrm{Hg}$ : black contrast, upper left corner; tip: dark gray contrast, lower right corner) shown in chronological order. Only the maximum values of each contact conductance are taken into account. The conductance values of new small nanocontacts (red circles) are calculated as the difference between two consecutive total conductance values of the $\mathrm{Hg}$ nanomenisci. These nanocontact conductance values are always either $0 G_{0}$ or of the order of $G_{0}$. In particular, image (d) proves that the conductance of a CNT in contact with the $\mathrm{Hg}$ droplet can be $0 G_{0}$. Also shown is a particle (diameter $>200 \mathrm{~nm}$ ), which does not make mechanical contact with the tip surface as it is at a different height. This particle is floating on the $\mathrm{Hg}$ surface (thick blue arrow) and has been most likely detached from the tip surface by a $\mathrm{Hg}$ immersion.
In the original paper of Frank et al. [18], Fig. 1 shows only a TEM image of the CNT-carrying tip whereas Fig. 2 shows conductivity-length dependence curves acquired in an ex situ scanning probe microscope (SPM) experiment without visualization (thus without certainty that it is the conductance of CNTs which is measured). This equally holds true for the subsequent papers $[28,34,38,39]$. In the work of Wang et al. [38], in situ experiments have been performed but any conductivity-length dependence curves possibly acquired in these experiments are not shown. Figure 3 of Wang et al. [38] shows an image of an in situ TEM transport experiment (however without a conductivity-length dependence) whereas Fig. 4 of Wang et al. [38] shows the conductivity-length dependence acquired in an independent ex situ atomic force microscope (AFM) experiment (without visualization, thus without certainty that it is the conductance of CNTs which is measured). In the work of Poncharal et al. [34], Fig. 1 shows a conductivity-length dependence curve acquired in an in situ experiment; however TEM images possibly acquired simultaneously with these electrical measurements are not shown, and thus it remains impossible to ascertain whether these conductance can be attributed to CNTs or not. In a subsequent paper of Poncharal et al. [28], Fig. 1(a) shows an image of an in situ TEM transport experiment (however without a conductivity-length dependence) whereas Fig. 1(b) shows the conductivity-length dependence acquired in an independent ex situ SPM experiment (without visualization, thus without certainty that it is the conductance of CNTs which is measured). Further in the same paper of Poncharal et al. [28], Figs. 2(d) and 2(e) only show TEM images of the CNTcarrying tip before and after transport measurements whereas Figs. 2(a)-2(c) show conductance-length dependence curves acquired in an ex situ SPM experiment (without visualization, thus again without certainty that it is the conductance of CNTs which is measured). It is true that Fig. 2(e) confirms that a small number of CNTs (or small CNT bundles which cannot be decided due to the insufficient magnification and resolution of the TEM image) out of a high initial number of CNTs or CNT bundles has survived the cyclic immersion process. Nonetheless, this fact alone is not sufficient to confirm that the conductance plateaus in Fig. 2(c) can really be attributed to the CNTs. Rather, it is much more likely that these conductance plateaus are due to $\mathrm{Hg}$ menisci as we argue in this paper. Poncharal et al. [28] even support our argument by showing a TEM image of a $\mathrm{Hg}$ meniscus sticking to the end of a CNT in Fig. 1(a). Further in the same paper of Poncharal et al. [28], Figs. 3-6 show conductance measurements from ex situ SPM experiments (without visualization), Fig. 7 in situ TEM observations without conductance-length dependence curves, 
(a)

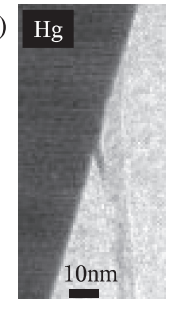

$\mathrm{t}=0 \mathrm{~s}$

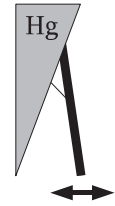

(b)

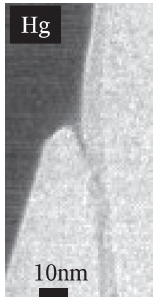

$\mathrm{t}=2 \mathrm{~s}$

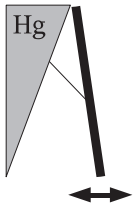

(c) $\mathrm{Hg}$

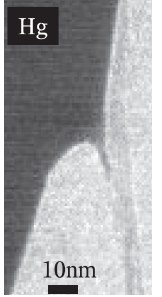

$\mathrm{t}=4 \mathrm{~s}$

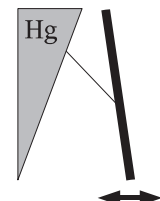

(d)

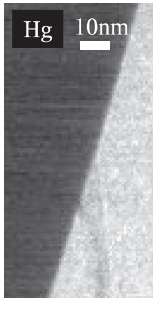

$\mathrm{t}=4 \mathrm{~s}$

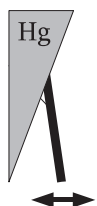

FIG. 7. A CNT rope which bifurcates close to the Hg surface. A thin part of the rope is in contact with a $\mathrm{Hg}$ meniscus which appears to be strongly pinned to it and which follows the CNT rope during its retraction from and approach to the $\mathrm{Hg}$ droplet of about $30 \mathrm{~nm}$. However, the majority of the CNTs inside the rope lie down on the $\mathrm{Hg}$ surface.

and Fig. 11 conductance value histograms from experiments on gold nanowires and not from experiments on CNTs. Another paper using the LMC method (Berger et al. [39]) does not show any conductance-length dependence curves at all, either from in situ or ex situ experiments. In addition to the above remarks, no conductance-length dependence curves corresponding to cyclic immersion in situ TEM experiments and no corresponding conductance value histograms are shown in any of the above papers [28,34,38,39].

As the nonexistence of successful conductance-length dependence measurements by the in situ LMC method does not exclude that such an experiment might be in principle possible, we chose a different approach in order to investigate this question: in order to investigate whether the in situ LMC method is suited to measure the conductance-length dependence of CNTs in the configuration depicted in Fig. 1(b2) if one manages to rule out the possibility of the removal of the CNTs from the tip surface and the measurement of the $\mathrm{Hg}$ nanomenisci and nanocontacts (instead of the CNT) in a specially designed in situ experiment, we have performed numerical simulations based on an analytical mechanical model of the CNT-Hg immersion process.

The analytical model (published separately [40]) assumes that a mobile contact on the CNT can be created in two ways: either by the CNT penetrating the Hg surface or by the CNT lying down on the $\mathrm{Hg}$ surface (see Fig. 7 for an example). Simulations based on the above analytical model show that most CNTs will not create a mobile contact with the $\mathrm{Hg}$ surface under realistic experimental conditions. (Especially, the most interesting objects, SWNTs and DWNTs with micrometric lengths and arbitrary diameters, will not create a mobile contact with the $\mathrm{Hg}$ even for experimentally unattainably small deviation angles.) In addition, the small minority of CNTs which will create a mobile contact will not behave as expected either: From the moment at which the tip of the CNT touches the $\mathrm{Hg}$ surface until the moment at which the tip of the CNT moves under the Hg surface or lies down on it, the
CNT-carrying sample will have to be approached by a certain distance to the $\mathrm{Hg}$ surface. This distance will be a systematic error on the electronic mean free path (EMFP) of the CNTs one tries to determine by these experiments and can be up to $100 \%$ of the measured value.

\section{CONCLUSION}

In conclusion, our experimental results show that the in situ LMC method (i.e., performed inside a TEM and thus with visualization) is well suited to create low-resistance contacts with the tip of the CNT in the configuration depicted in Fig. 1(b1) and therefore also to measure the conductance of the whole CNT. However, our in situ experiments also prove that the ballistic and quantum conductance measured by the ex situ LMC method (i.e., not performed in a TEM and thus without visualization) in both configurations (b1) and (b2) depicted in Fig. 1 is likely to give false positives for three reasons: (a) the CNTs are likely to be removed from the tip surface through contact with the $\mathrm{Hg}$, (b) occurring $\mathrm{Hg}$ /tip-surface nanocontacts are likely to be mistaken for quantum-conductor CNTs, and (c) occurring $\mathrm{Hg}$ nanomenisci are likely to be mistaken for ballistic-conductor CNTs. As these possibilities cannot be ruled out in an ex situ LMC experiment, the results obtained by this method have to be interpreted with great caution keeping in mind that they are very likely measurements on other objects than CNTs. (It is also for this reason that only the in situ LMC method - and not the ex situ LMC method-is suited to measure the conductance of the whole CNT.) In consequence, many previous experimental results claiming ballistic and quantum conductance in CNTs have to be reinterpreted.

In situ experiments and numerical simulations based on an analytical mechanical model of the CNT-Hg immersion process (published separately [40]) show that not only the ex situ but also the in situ LMC method is unsuitable for ballistic- and quantum-conductance measurements on CNTs in the configuration depicted in Fig. 1(b2). The reason is that most CNTs will not create a mobile contact with the $\mathrm{Hg}$ surface under realistic experimental conditions, and that the rest of the CNTs will have a systematic error on the measurement of its mean free path of up to $100 \%$ of the measured value.

Finally, our experiments have shown that Hg-tip nanocontacts between a $\mathrm{Hg}$ droplet and a tip covered by a CCVD residue layer of disordered graphitic and metallic particles can have a conductance close to the conductance quantum $G_{0}$ which cannot be explained either by a simple classical or by a simple quantum model. Their conductance values are reproducible and depend on the precise location at which the $\mathrm{Hg}$ nanomeniscus touches the tip surface. A possible explanation could be an inhomogeneous conductivity of the CCVD residue layer leading to a percolation-type conductance.

In conclusion, these experimental results show that the $e x$ situ LMC method is not reliable for ballistic- and quantumconductance measurements on CNTs. This work challenges previously published and accepted results and we hope that it will help to resolve the controversy about the ballistic and diffusive nature of CNTs. 
[1] S. Iijima, Nature (London) 354, 56 (1991).

[2] R. Saito, G. Dresselhaus, and M. Dresselhaus, Physical Properties of Carbon Nanotubes (Imperial College Press, London, 1998).

[3] W. Hoenlein, G. S. Duesberg, A. P. Graham, F. Kreupl, M. Liebau, W. Pamler, R. Seidel, and E. Unger, Microelectron. Eng. 83, 619 (2006).

[4] M. M. Shulaker, G. Hills, N. Patil, H. Wei, H.-Y. Chen, H. S. Philip Wong, and S. Mitra, Nature (London) 501, 526 (2013).

[5] D. E. Nikonov and I. A. Young, Proc. IEEE 101, 2498 (2013).

[6] D. E. Nikonov and I. A. Young, IEEE Int. Electron Devices Meet. 2012, 25.4.1.

[7] A. D. Franklin, M. Luisier, S.-J. Han, G. Tulevski, C. M. Breslin, L. Gignac, M. S. Lundstrom, and W. Haensch, Nano Lett. 12, 758 (2012).

[8] L. Wei, D. J. Frank, L. Chang, and H.-S. P. Wong, IEEE Int. Electron Devices Meet. 2009, 1.

[9] R. Martel, T. Schmidt, H. R. Shea, T. Hertel, and P. Avouris, Appl. Phys. Lett. 73, 2447 (1998).

[10] S. J. Tans, A. R. M. Verschueren, and C. Dekker, Nature (London) 393, 49 (1998).

[11] A. Bachtold, P. Hadley, T. Nakanishi, and C. Dekker, Science 294, 1317 (2001).

[12] Z. H. Chen, J. Appenzeller, Y. M. Lin, J. Sippel-Oakley, A. G. Rinzler, J. Y. Tang, S. J. Wind, P. M. Solomon, and P. Avouris, Science 311, 1735 (2006).

[13] Q. Cao, H.-s. Kim, N. Pimparkar, J. P. Kulkarni, C. Wang, M. Shim, K. Roy, M. A. Alam, and J. A. Rogers, Nature (London) 454, 495 (2008).

[14] N. Patil, A. Lin, J. J. Zhang, H. Wei, K. Anderson, H. S. P. Wong, and S. Mitra, IEEE Trans. Nanotechnol. 10, 744 (2011).

[15] Q. Cao, S.-j. Han, G. S. Tulevski, Y. Zhu, D. D. Lu, and W. Haensch, Nat. Nanotechnol. 8, 180 (2013).

[16] J. Zhang, A. Lin, N. Patil, H. Wei, L. Wei, H. S. P. Wong, and S. Mitra, IEEE Trans. CAD 31, 453 (2012).

[17] M. M. Shulaker, H. Wei, N. Patil, J. Provine, H.-Y. Chen, H. S. P. Wong, and S. Mitra, Nano Lett. 11, 1881 (2011).

[18] S. Frank, P. Poncharal, Z. L. Wang, and W. A. de Heer, Science 280, 1744 (1998).

[19] A. Urbina, I. Echeverria, A. Perez-Garrido, A. Diaz-Sanchez, and J. Abellan, Phys. Rev. Lett. 90, 106603 (2003).

[20] L. Langer, V. Bayot, E. Grivei, J. P. Issi, J. P. Heremans, C. H. Olk, L. Stockman, C. Van Haesendonck, and Y. Bruynseraede, Phys. Rev. Lett. 76, 479 (1996).
[21] A. Bachtold, C. Strunk, J. P. Salvetat, J. M. Bonard, L. Forro, T. Nussbaumer, and C. Schonenberger, Nature (London) 397, 673 (1999).

[22] H. Kajiura, H. J. Huang, and A. Bezryadin, Chem. Phys. Lett. 398, 476 (2004).

[23] H. Kajiura, A. Nandyala, U. C. Coskun, A. Bezryadin, M. Shiraishi, and M. Ata, Appl. Phys. Lett. 86, 122106 (2005).

[24] H. Kajiura, A. Nandyala, and A. Bezryadin, Carbon 43, 1317 (2005).

[25] N. R. Wilson, D. H. Cobden, and J. V. Macpherson, J. Phys. Chem. B 106, 13102 (2002).

[26] J. Y. Chen, A. Kutana, C. P. Collier, and K. P. Giapis, Science 310, 1480 (2005).

[27] M. Kociak, K. Hirahara, K. Suenaga, and S. Iijima, Eur. Phys. J. B 32, 457 (2003).

[28] P. Poncharal, C. Berger, Y. Yi, Z. L. Wang, and W. A. de Heer, J. Phys. Chem. B 106, 12104 (2002).

[29] L. Marty, A. Iaia, M. Faucher, V. Bouchiat, C. Naud, M. Chaumont, T. Fournier, and A. M. Bonnot, Thin Solid Films 501, 299 (2006).

[30] Y. A. Kasumov, A. Shailos, I. I. Khodos, V. T. Volkov, V. I. Levashov, V. N. Matveev, S. Gueron, M. Kobylko, M. Kociak, H. Bouchiat, V. Agache, A. S. Rollier, L. Buchaillot, A. M. Bonnot, and A. Y. Kasumov, Appl. Phys. A 88, 687 (2007).

[31] Nanofactory Instruments AB, Göteborg, Sweden.

[32] K. Svensson, Y. Jompol, H. Olin, and E. Olsson, Rev. Sci. Instrum. 74, 4945 (2003).

[33] N. Agrait, A. L. Yeyati, and J. M. van Ruitenbeek, Phys. Rep. 377, 81 (2003).

[34] P. Poncharal, S. Frank, Z. L. Wang, and W. A. de Heer, Eur Phys. J. D 9, 77 (1999).

[35] See Supplemental Material at http://link.aps.org/supplemental/ 10.1103/PhysRevB.90.195431 for the illustration of $\mathrm{Hg}$ immersion-induced modifications of the CNTs on the apex of a metalized tip.

[36] C. Kittel, Introduction to Solid State Physics (Wiley and Sons, Hoboken, NJ, 2004).

[37] S. Datta, Electronic Transport in Mesoscopic Systems (Cambridge University Press, Cambridge, UK, 1995).

[38] Z. L. Wang, P. Poncharal, and W. A. de Heer, J. Phys. Chem. Solids 61, 1025 (2000).

[39] C. Berger, P. Poncharal, Y. Yi, and W. de Heer, J. Nanosci. Nanotechnol. 3, 171 (2003).

[40] M. Kobylko, Phys. Rev. B 90, 195432 (2014). 